\title{
Viscoelastic Depinning of Driven Systems: Mean-Field Plastic Scallops
}

\author{
M. Cristina Marchetti, A. Alan Middleton, and Thomas Prellberg \\ Physics Department, Syracuse University, Syracuse, NY 13244
}

(December 23, 1999)

\begin{abstract}
We have investigated the mean field dynamics of an overdamped viscoelastic medium driven through quenched disorder. The model allows for the coexistence of pinned and sliding regions and can exhibit continuous elastic depinning or first order hysteretic depinning. Numerical simulations indicate mean field instabilities that correspond to macroscopic stick-slip events and lead to premature switching. The model describes the elastic and plastic dynamics of driven vortex arrays in superconductors and other extended disordered systems.
\end{abstract}

Extended condensed matter systems driven over quenched disorder exhibit a very complex dynamics, including nonequilibrium phase transitions and history dependence. Such systems include vortex arrays in type-II superconductors [1], charge density waves in anisotropic conductors [2, 3], and many others. Closely related behavior also arises in friction and lubrication [4], where a surface or monolayer is brought in contact with another solid surface and forced to slide relative to it.

Most of the theoretical work to date has focused on the dissipative dynamics of driven elastic media that are distorted by disorder, but cannot tear. At zero temperature such systems exhibit a sharp depinning transition from a pinned to a sliding state [3,5]. The transition, first studied in the context of charge density waves, is continuous, with universal critical behavior. The sliding state is unique and there is no hysteresis or history dependence [6]. More recent work, while still focusing on elastic media, has shown that the dynamics is quite rich well into the uniformly sliding state [7 11].

On the other hand, experiments 12,13 and simulations [14,15] show that the elastic medium model is inadequate for many physical systems with strong disorder that upon depinning exhibit a spatially inhomogeneous plastic response, without long wavelength elastic restoring forces. In this plastic flow regime, topological defects proliferate and the system is broken up in fluid-like regions flowing around pinned solid regions. Not much progress has been made in describing this behavior analytically. The wealth of experimental work on driven vortex arrays clearly indicates that, in most of the field and temperature region of interest, the current-driven vortex dynamics is strongly history dependent, with long-term memory and switching as the system explores a variety of nonequilibrium sliding states 12, 13].

In this paper we describe a coarse-grained model for the dynamics of a driven medium that allows for spatially inhomogeneous response, with the coexistence of moving and pinned regions. The model is inspired by the wellknown phenomenology of viscoelasticity in dense fluids [16]. The elastic couplings between the local displace- ments are replaced by couplings that are nonlocal in time and allow for elastic restoring forces to turn into dissipative fluid flow on short time scales. The model yields elastic depinning in one limit; as the parameters are varied, it incorporates continuous depinning, hysteretic plastic depinning and eventually viscous flow, allowing the crossovers between these regimes (such as those, observed in vortex arrays $[12,15])$ to be studied in detail. For a wide range of parameter values the depinning transition is first order, with velocity hysteresis (switching.) The nonlinear velocity-force characteristic can be evaluated analytically in mean field for various types of pinning forces, under the assumption of constant mean field velocity. Numerical simulations confirm the inhomogeneous nature of the dynamics, with pinning and tearing (coexisting moving and pinned degrees of freedom.) In addition, the mean velocity near depinning fluctuates, due to macroscopic stick-slip type events. These events appear to only mildly violate the uniform mean-velocity assumption but directly lead to switching from one velocity branch to another before the first branch terminates (premature switching.) Models that account for switching in charge density waves and are in spirit similar to ours have been proposed and studied by Strogatz and collaborators [17]. In such models, plasticity is modeled by a non-convex elastic potential, in contrast with the velocity convolutions studied here. A model similar to ours has also been proposed for crack propagation [18].

The model: a driven viscoelastic medium. To motivate our model, we first recall the generic model of driven elastic media [3] discussed extensively in the literature, where the long-wavelength dynamics is described in terms of a coarse-grained displacement field, $u(\mathbf{r}, t)$. The displacement fields represent deformations of regions pinned collectively by disorder (e.g., a Larkin domains) and are coupled by convex elastic interactions. No topological defects are allowed. Considering, for simplicity, the overdamped dynamics of a scalar field (the model is easily extended to the more general case) and modeling the displacement field on lattice sites, $u(\mathbf{r}, t) \rightarrow u_{i}(t)$, the equation of motion for the local field $u_{i}$ (measured in the 
laboratory frame [19]) at site $i$ is

$$
\gamma_{0} \dot{u}_{i}=\sum_{\langle i j\rangle} \mu_{i j}\left(u_{j}-u_{i}\right)+F+F_{i}\left(u_{i}\right)
$$

where the summation is restricted to nearest neighbor pairs and $\gamma_{0}$ is the friction. If all the nearest-neighbor elastic couplings, $\mu_{i j} \geq 0$, are equal, the first term on the right hand side of Eq. (11) is the discrete Laplacian in $d$ dimensions. The second term is the homogeneous driving force, $F$, and $F_{i}\left(u_{i}\right)$ denotes the pinning force arising from a quenched random potential, $V_{i}\left(u_{i}\right)$, $F_{i}\left(u_{i}\right)=-d V_{i} / d u_{i}=h_{i} f\left(u_{i}-\beta_{i}\right)$, with $f(u)$ a periodic function with period 1 and $\beta_{i}$ random phases uniformly distributed in $[0,1]$. The $h_{i}$ are chosen independently at each site from a distribution $\rho(h)$. One of the quantities of interest is the average velocity of the driven medium, $\bar{v}(F)=N^{-1} \sum \dot{u}_{i}$. For an elastic medium there is a unique stationary sliding state for $F>F_{c}$, with critical behavior $\bar{v}(F) \sim\left(F-F_{c}\right)^{\beta}[3]$, and no hysteresis at the transition [6].

We now modify the elastic interactions in Eq. (1) to allow for local tearing of the medium. Inspired by standard models of viscoelasticity, we replace the elastic interaction by couplings to the local velocity field, $v_{i}=\dot{u}_{i}$, that are nonlocal in time. Our model equation for the overdamped dynamics of a driven viscoelastic medium is 20

$\gamma_{0} \dot{u}_{i}=\sum_{\langle i j\rangle} \int_{0}^{t} d s C_{i j}(t-s)\left[\dot{u}_{j}(s)-\dot{u}_{i}(s)\right]+F+F_{i}\left(u_{i}\right)$,

where the viscous couplings $C_{i j}(s)$ have finite first moments, $\int_{0}^{\infty} d s C_{i j}(s)=\eta_{i j}<\infty$ and $C_{i j}(0)=\mu_{i j}$. Such nonlocal couplings to velocity are of course not present at the microscopic level, but are generated generically upon coarse-graining [16,21]. Eq. (2) is a coarse-grained model for the dynamics of a driven disordered medium that allows for slip or friction of the interacting Larkin domains relative to each other.

A simple, yet successful, model of viscoelasticity due to Maxwell is obtained when the memory kernels are assumed to be uniform in space and to decay exponentially in time, according to $C_{i j}(t)=\mu e^{-t / \tau}$, with $\tau=\eta / \mu$ the Maxwell relaxation time. For $\tau \rightarrow \infty$ and fixed $\mu$, Eq. (2) reduces to Eq. (1) for a driven elastic medium. For $\tau \rightarrow 0$ and $\eta$ fixed, the first term on the right hand side of Eq. (2) can be approximated as $\eta \sum_{\langle i j\rangle}\left[v_{j}(t)-v_{i}(t)\right]$, which represents viscous forces coupling the local fluid velocity at different spatial points. In this limit, Eq. (2) describes an overdamped lattice-fluid of viscosity $\eta$. We propose Eq. (2) as a simple, yet realistic model for a driven disordered system that exhibits spatially inhomogeneous plastic response.
Mean Field Approximation. As for the driven elastic media, substantial analytical progress in two or three dimensions is presumably only possible via perturbation theory or by a functional renormalization group treatment 22]. An alternative approach that has provided valuable insight for a driven elastic medium is mean field theory (MFT), first discussed by D. S. Fisher [3]. MFT is formally valid in the limit of infinite-range interaction, with $\sum_{j} C_{i j}=N C(t)$ held fixed. The equation of motion for the displacement at each site is then given by

$$
\gamma_{0} \dot{u}_{i}=\int_{0}^{t} y d s C(t-s)\left[\bar{v}(s)-\dot{u}_{i}(s)\right]+F+F_{i}\left(u_{i}\right),
$$

where the mean field is given by $\bar{u}(t)=N^{-1} \sum_{i=1}^{N} u_{i}(t)$, and $\bar{v}(t)=\dot{\bar{u}}(t)$.

If the memory kernel $C(t)$ is chosen to be of the Maxwell form, it is then possible to transform the integrodifferential equation (3) to a second-order differential equation, given by

$$
\tau \ddot{u}+\gamma\left(\eta, \tau, h ; u_{i}\right) \dot{u}=F+F_{i}\left(u_{i}\right)+\eta \bar{v},
$$

with $\gamma\left(\eta, \tau, u_{i} ; h\right)=1+\eta-\tau \frac{\partial F_{i}}{\partial u_{i}}$ an effective friction. We have scaled Eq. (4) by letting $\tau \rightarrow \tau h_{0}, t \rightarrow t h_{0}$, $\eta \rightarrow \eta / \gamma_{0}, F \rightarrow F /\left(\gamma_{0} h_{0}\right)$ and $h \rightarrow h /\left(\gamma_{0} h_{0}\right)$, where $h_{0}$ is the characteristic scale of the distribution $\rho(h)$. With this change of variables, the model is now characterized by two parameters, $\eta$ and $\tau$, and the shape of $\rho(h)$. The MF equation for our viscoelastic model closely resembles the MF equation for a driven massive elastic medium, with $\tau$ playing the role of the mass. The most important difference is that in the massive elastic medium the MF term $\eta \bar{v}$ is replaced by $\mu \bar{u}$. As a result, the MFT of a driven massive elastic medium even with constant $\bar{v}$ contains three degrees of freedom (as opposed to the two of our problem) and the dynamics of a single $u_{i}$ is chaotic 26,27.

We are first interested here in steadily sliding solutions of the MF model, Eq. (3). It is natural to look for periodic solutions $u_{p}(t ; h)$ of period $T(h),\left(\int_{0}^{T(h)} d t \dot{u}_{p}(t ; h)=\right.$ 1) that may set in after an initial transient $(t \gg T(h))$. Such solutions need not be unique. Guided by a large body of previous work on driven elastic media, we focus on the MFT for the case of a pinning potential with cusplike singularities, which better captures the physics of the corresponding finite-dimensional model [23. An explicit solution of Eq. (4) can be obtained for the scalloped parabolic potential, $V(u)=(h / 2)\left(u^{2}-u+1 / 4\right)$. In this case Eq. (4) is linear and its general solution is $u_{p}(t ; h)=$ $C_{1} \exp \left(-\lambda_{1} t\right)+C_{2} \exp \left(-\lambda_{2} t\right)+1 / 2+(\eta \bar{v}+F) / h$, with $\lambda_{1,2}=\left(1+\eta+\tau h \pm \sqrt{(1+\eta+\tau h)^{2}-4 \tau h}\right) /(2 \tau)$. For each fixed value of $h$, we obtain an implicit equation for the period, $T(h), \eta \bar{v}+F=\mathcal{G}(T ; \eta, \tau, h)$, with 


$$
\mathcal{G}(T ; \eta, \tau, h)=\frac{\lambda_{1}\left(1-e^{-\lambda_{1} T}\right)-\lambda_{2}\left(1-e^{-\lambda_{2} T}\right)+\tau \lambda_{1} \lambda_{2}\left(e^{-\lambda_{1} T}-e^{-\lambda_{2} T}\right)}{\left(\lambda_{1}-\lambda_{2}\right)\left(1-e^{-\lambda_{1} T}\right)\left(1-e^{-\lambda_{2} T}\right)}-\frac{h}{2} .
$$

The solution of Eq. (5), together with the self-consistency constraint $\bar{v}=<[T(h)]^{-1}>_{h}$, determines the drift velocity as a function of driving force, $F$. When $T\left(h_{i}\right) \rightarrow \infty$, the $u_{i}$ is pinned.

Figure 1 shows the analytical solution for the mean velocity as a function of driving force for $\rho(h)=\exp (-h)$. The depinning occurs at $F=0$ for all distributions of pinning strengths, $\rho(h)$, with support not bounded from below by a positive $h_{\min }$. For small $\eta$ and $\tau$, corresponding to weak coupling among the local displacements, the analytical solution is single-valued and the depinning is continuous. For large $\eta$ and $\tau$ the analytical solution yields multi-valued velocity curves, reflecting the existence of multiple sliding states, and the depinning is hysteretic. As shown in the inset of Fig. 1, there is a critical value, $\eta_{c}(\tau)$, that separates single-valued from multivalued solutions. The value $\eta=\eta_{c}$ is a critical point and the velocity curve is expected to exhibit critical scaling. While the value of $\eta_{c}$ depends on $\tau$, the existence of an hysteretic region at large $\eta$, with coexistence of sliding and moving states and early switching (see also Fig. 1) occurs for all finite values of $\tau$, including $\tau=0$. For $\tau \rightarrow \infty$ and $\eta \rightarrow \infty$, with the ratio $\mu=\eta / \tau$ held fixed, Eq. (4) reduces to the MFT of an overdamped elastic medium 24]. In this case an analytical solution is available and the velocity vanishes linearly as $F \rightarrow F_{c}$ [25].

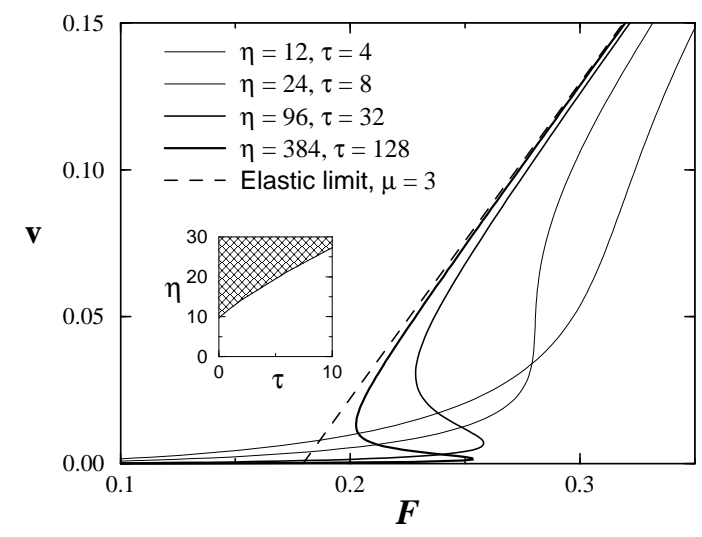

FIG. 1. Analytical MFT velocity versus force curve for $\rho(h)=\exp (-h)$ and selected values of $\tau$ and $\eta$ along the path $\eta=3 \tau$ in parameter space. As $\tau$ and $\eta$ increase along this path, the $v(F)$ curve becomes hysteretic. The dashed line is the result for the purely elastic model with $\mu=3$, showing the convergence of the viscoelastic model to the elastic model for large $\tau$ and $\eta$. The inset shows the regions of parameters where depinning is hysteretic (shaded region) and where it is continuous (unshaded region.)

Numerical work. We have investigated the stability of the branches of the analytically determined current-drive relationship. We performed direct numerical simulation of the equations of motion, for both force drive and constrained mean velocity. The simulations were performed using two codes, for verification: a Runge-Kutta integration and an event-driven Euler integration, with the "events" being crossings of a displacement $u_{i}$ from one parabolic region to the next. The results were checked for insensitivity to time step $\Delta t$ and size $N$. For the constant $\bar{v}$ constraint, the drive-velocity relationship matches the analytical prediction.

In the regions where the velocity is a unique function of the drive, the simulation results with slowly changing $F$ for the force-drive curve match very closely those of the analytical results, which assume a constant $\bar{v}$. In the presumed hysteretic region, though, the simulation results can be quite different. In particular, we note two features: mean field velocity oscillations on the lower branch and "early" switching, where the mean velocity switches from the lower to upper branch prior to the end of the analytically computed hysteresis region. A sample hysteresis curve indicating early switching is shown in Fig. 2. We have computed the magnitude of the fluctuations in the mean velocity on the upper branch as a function of $N$ : the results are numerically consistent with a magnitude $\propto N^{-1 / 2}$, indicating that these fluctuations vanish as $N \rightarrow \infty$. The fluctuations on the lower branch do not vanish in the limit of large $N$, however. These fluctuations are presumably due to an instability of the constant $\bar{v}$ solution in the large volume limit. We hypothesize, with the support of detailed analysis of the numerics, that nearly depinned degrees of freedom (which would remain pinned at constant $\bar{v}$ ) are made unstable by velocity fluctuations and lead to an avalanche type of behavior, which causes a peak in $\bar{v}$. The magnitude of this instability apparently becomes large enough to drive the mean velocity to the upper branch before the presumed constant $\bar{v}$ velocity jump occurs.

In conclusion, we have introduced a coarse-grained model of plastic flow that allows for slip of coherently pinned domains. We have solved this model analytically in mean field for the case of Maxwellian kernel, under the assumption of non-fluctuating mean velocity. We find that (1) the model exhibits both continuous and first order hysteretic depinning as the parameters are varied, (2) we can recover the case of elastic depinning in one limit, (3) pinned and sliding regions coexist in the hysteretic regime, and (4) the mean velocity curves display features observed in experiments. Numerical simulations suggest that the behavior is much richer than suggested by the MF calculation and includes stick-slip-like instabilities which lead to early switching. Strong history dependence has been observed in the dc response of vortex 
lattices in type-II superconductors [12,13] and in charge density waves [28]. Hysteresis in vortex lattice motion is most pronounced in the region of the so-called peak effect, where the dc response during ramp-up of the current proceeds via a series of jumps. These have been attributed to strong spatial inhomogeneities in the distribution of vortex velocities, not unlike what is observed in our model [29]. We expect that in finite dimensions, the transition to hysteresis will be characterized by nontrivial universal scaling exponents [30], similar to the situation for hysteresis in random magnets [22], and that these exponents could be experimentally tested.

One of us (MCM) thanks Daniel Fisher and Jennifer Schwarz for illuminating discussions. The work was supported by NSF through grants DMR-9730678, powredmr 9805818 and career-dmr9702242.

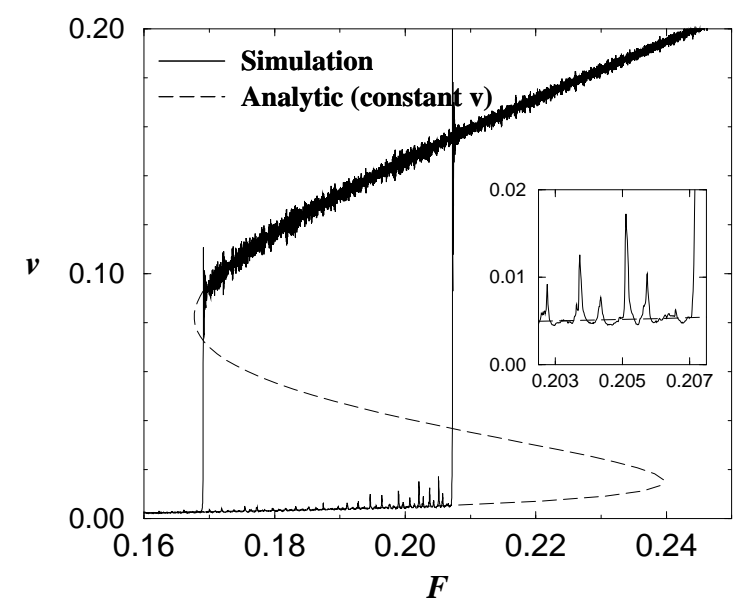

FIG. 2. Comparison of direct numerical simulation (solid line) with analytic predictions (dashed line), which assume a constant $\bar{v}$, for $\rho(h)=\exp (-h), \eta=32, \tau=0.8, N=16384$, and a ramp rate of $d F / d t=2.5 \times 10^{-6}$. The field $F$ is cyclic in time. The results are in near exact agreement for much of the history. In the hysteretic region, on the lower branch, the mean field velocity occasionally spikes due to macroscopic events.

[1] G. Blatter et al., Rev Mod. Phys. 66, 1125 (1994).

[2] G. Grüner, Rev. Mod. Phys. 60, 1129 (1988).

[3] D.S. Fisher, Phys. Rev. B 31, 1396 (1985).

[4] B.N.J. Persson, Sliding Friction: Physical principles and Applications (Springer, Heidelberg, 1998).

[5] A.A. Middleton, Ph.D. thesis, Princeton University, 1990; A.A. Middleton and D.S. Fisher, Phys. Rev. Lett. 66, 92 (1991).

[6] A.A. Middleton, Phys. Rev. Lett. 68, 670 (1992).

[7] D.S. Fisher, K. Dahmen, S. Ramanathan, and Y. BenZion, Phys. Rev. Lett. 78, 4885 (1997).
[8] T. Giamarchi and P. Le Doussal, Phys. Rev. Lett. 76, 3408 (1996).

[9] L. Balents, M.C. Marchetti and L. Radzihovsky, Phys. Rev. B 57, 7705 (1998).

[10] K. Moon, R.T. Scalettar, and G.T. Zimányi, Phys. Rev. Lett. 77, 2278 (1996).

[11] F. Pardo et al., Nature 396, 348 (1998); and references therein.

[12] S. Bhattacharya and M.J. Higgins, Phys. Rev. Lett. 70, 2617 (1993); M.J. Higgins and S. Bhattacharya , Physica C 257, 232 (1996).

[13] W. Henderson, et al., Phys. Rev. Lett. 80, 381 (1998).

[14] A.-C. Shi and J. Berlinsky, Phys. Rev. Lett., 67, 1926 (1991).

[15] M.C. Faleski, M.C. Marchetti and A. A. Middleton, Phys. Rev. B 54, 12427 (1996).

[16] J.P. Boon and S. Yip, Molecular Hydrodynamics (Dover, 1992).

[17] S.H. Strogatz et al., Phys. Rev. Lett. 61, 2380 (1988); S.H. Strogatz and R.M. Westervelt, Phys. Rev. B 40, 10501 (1989).

[18] J. Schwartz and D. S. Fisher (unpublished).

[19] We neglect here convective derivatives, which may indeed be important near a first order transition.

[20] The disorder $\beta_{i}$ can be absorbed into the initial conditions for $u_{i}$, as the coupling is only through $\dot{u}_{i}$. Nonuniform initial conditions should then be chosen to reflect this disorder, otherwise degenerate solutions arise.

[21] M.C. Marchetti, J. Applied Phys. 69, 5185 (1991).

[22] The study of the hysteresis of the random field Ising magnet may be applicable; see, e.g., O. Perkovic, K. A. Dahmen, and James P. Sethna, Phys. Rev. Lett. 75, 4528 (1995).

[23] Numerical simulations [5] and functional RG treatments 25. have shown that in low dimensions the critical region exhibits a jump-dominated jerky dynamics even when the pinning potential is smooth on short scales. In MFT, where the force is averaged over many neighbors, this crucial feature of the dynamics survives only for pinning potentials with cusp-like singularities.

[24] The fact that the strictly elastic limit is obtained only at a singular point in parameter space is an attractive feature of our model. It is consistent with the observation by S.N. Coppersmith [Phys. Rev. Lett. 65, 1044 (1990)] that unbounded strains, yielding topological defects, are always generated in an elastic medium of infinite extent driven by a uniform force over quenched disorder.

[25] O. Narayan and D.S. Fisher, Phys. Rev. B 46, 11520 (1992).

[26] O.M. Braun et al., Phys. Rev. B 54, 321 (1996); O.M. Braun, A.R. Bishop, and J. Röder, Phys. Rev. Lett. 79, 3692 (1997).

[27] T. Strunz and F.-J. Elmer, Phys. Rev. E 58, 1601 (1998); 58, 1612 .

[28] S. G. Lemay, R. E. Thorne, Y. Li, and J. D. Brock, Phys. Rev. Lett. 83, 2793 (1999).

[29] In finite dimensions, a conservation law would be added to most properly describe vortex motion, while this is not necessary for the charge density waves condensate.

[30] In preparation, M. C. Marchetti, A. A. Middleton, and T. Prellberg. 